\title{
MedienPädagogik
}

Zeitschrift für Theorie und Praxis der Medienbildung

\section{Mit Open-Source-Software die Lehre öffnen - ein Plädoyer}

Oliver Tacke

\begin{abstract}
Zusammenfassung
Die technische Infrastruktur, die zum Erstellen, Teilen und Remixen von freien Bildungsmaterialien genutzt wird, kann erheblichen Einfluss auf die Gestaltung derselben haben und verdient eine stärkere Betrachtung als bisher. Der «Action Plan» des zweiten OERWeltkongresses scheint diese Aussage zu stützen. So werden in ihm an mehreren Stellen zu bearbeitende Felder genannt werden, die direkt oder indirekt von der technischen Infrastruktur abhängen - ohne sie jedoch selbst zum Ziel von Bemühungen zu machen. Die Infrastruktur ist jedoch nicht lediglich ein Werkzeug, das didaktischen Überlegungen Wirkung verleiht, sondern gleichsam unterschwelliges Vehikel für zu transportierende Werte. Da Open-Source-Software viele Werte mit der OER-Bewegung teilt, sollte ihr trotz der immer wieder auftretenden Unbequemlichkeiten im Vergleich zu «unfreier) Software der Vorzug gegeben werden. Der Beitrag versteht sich als Plädoyer, das exemplarisch anhand der offenen Software H5P zeigt, wie sich mit ihr zahlreiche Tätigkeiten unterstützen lassen, die der Öffnung der Lehre dienen. Ferner wird argumentiert, dass die Kongruenz der Werte von offener Bildung und offener Software mehr ist als reine Ästhetik, sondern im besten Fall zur Verbreitung dieser Werte beitragen kann.
\end{abstract}

\section{A plea for opening education with open source software}

\begin{abstract}
The technological infrastructure that is used for creating, sharing and remixing open educational resources can influence their design considerably. The infrastructure therefore deserves more attention than before. The "action plan» of the second OER world congress seems to support this claim. It mentions several actions that should be taken, and many of them directly or indirectly rely on technical infrastructure - however, it doesn't mention actions related to the technical infrastructure itself. Still, it is more than just a tool that implements pedagogical thoughts. It can also be a vehicle that transports its underlying values. Since open source software shares many values with open education, it should take precedence over closed software. This article pleads to do so and uses the open software H5P as an example to demonstrate how it supports many actions that are intended to open education. Furthermore, the article arguments that the similarity of their values is more than just an aesthetic gimmick, but can even contribute to their diffusion.
\end{abstract}

Tacke, Oliver. 2018. «Mit Open-Source-Software die Lehre öffnen - ein Plädoyer». MedienPädagogik 32, (Oktober), 41-50. https://doi. org/10.21240/mpaed/32/2018.10.22.X. 


\section{Ist nicht schon offen, was Zugang schafft?}

Open Educational Resources gewinnen in Deutschland langsam an Fahrt. Dieser subjektive Eindruck entsteht jedenfalls mit Blick auf die vergangenen Jahre: Es gibt zahlreiche BarCamps und Konferenzen, auf denen sich die Anwesenden informieren und austauschen. Es gibt formale Weiterbildungsangebote und Onlinekurse, die zum Erweitern der eigenen Kenntnisse einladen. Und es gibt nicht zuletzt Menschen, die Inhalte unter einer offenen Lizenz zur Verfügung stellen und damit den OER-Pool vergrössern.

Die besten Inhalte nutzen jedoch wenig, wenn keine geeignete Infrastruktur zur Verfügung steht, um sie zu verwenden. Das ist nicht wesentlich anders als bei Autos. Für sie musste erst ein Netz an Tankstellen geschaffen werden, damit sie sich stark verbreiten konnten. «Es geht nicht nur darum, alle diese vielen Lehr- und Lernmaterialien zu erschaffen. [...] Alle Infrastrukturen müssen sich verändern» (Dueck 2009, 68). Für das Feld der freien Bildungsmaterialien bedeutet das, den Blick auf Hardund Software nicht aus dem Blick zu verlieren.

Vordergründig bieten kostenfrei nutzbare Plattformen bereits genügend Möglichkeiten, um Inhalte zu finden, zu erstellen, zu bearbeiten und mit anderen zu teilen. Softwaregigant Google etwa lädt dazu ein, seine Texte mit GoogleDocs (https://docs. google.com) zu verfassen und anderen zur Verfügung zu stellen. Das funktioniert hervorragend. Der Quelltext für GoogleDocs ist jedoch nicht offen verfügbar. Sollte Google irgendwann den Dienst einstellen, muss man hoffen, dass beim Exportieren in die Dateiformate anderer Programme nicht zu viele Details der Inhalte verloren gehen. Dass die Daten zudem unter der Kontrolle Fremder stehen und sich Datenschutzfragen auftun, sei nur erwähnt und nicht weiter ausgeführt.

Aus einer utilitaristischen Sicht spielt dies keine Rolle. Die Software funktioniert, bietet kaum praktische Zugangshürden und wird schon von vielen anderen genutzt. Geht es einzig um die Zweckorientierung, so ist die Parole "Offen ist, was Zugang schafft» (Muuß-Merholz 2017) durchweg nachvollziehbar. Ohne in einen Moralabsolutismus verfallen zu wollen (vgl. dazu auch die Unterscheidung von «(fast) offenen Inhalten» und «offenen Inhalten+» in Muuß-Merholz 2014), könnte man jedoch aus einer eher deontologischen Position heraus entgegnen, dass das Wesen des Weges bereits so beschaffen sein solle wie das Ziel selbst («Der Weg ist das Ziel»). Offenheit mit geschlossenen Mitteln erreichen zu wollen, verstiesse gegen dieses Ideal.

Denjenigen, die einer solchen Widersprüchlichkeit entgehen möchten, bleibt der Einsatz von offen lizenzierter Software (Open-Source-Software, kurz: OSS). Wie die in der OER-Szene bekannten Creative-Commons-Lizenzen räumt sie Nutzerinnnen und Nutzern umfassende Rechte ein. Je nach Wahl der Lizenz reicht auch hier die Bandbreite von der kostenfreien Nutzung bis hin zur kompletten Freigabe für Bearbeitung und Weitergabe. Die an OSS beteiligten Entwicklerinnen und Entwicklern verstehen diese Offenheit vielfach als normative Verpflichtung, die ethische, politische, 
technische und ökonomische Fragen nicht als losgelöst voneinander betrachtet (vgl. Stalder 2016, 186). Der Zweck allein heiligt nicht die Mittel.

In diesem Beitrag geht es anhand von Beispielen darum, welchen Beitrag OSS leisten kann, um Menschen beim Öffnen ihrer Lehre oder ihres Lernens zu unterstützen. Unter Rückgriff auf Euler und Poupart (2018) werden darunter fünf Kategorien von Merkmalen bzw. Tätigkeiten unterschieden:

1. Re-Usability: z.B. offene Standards und Lizenzen verwenden

2. Sharing: z.B. Inhalte auf Plattformen im Netz teilen

3. Collaboration: z.B. gemeinsam Inhalten erstellen oder annotieren

4. Discoverability: z.B. semantische Metadaten ergänzen oder in Suchmaschinen einbinden

5. Outreach: z.B. über soziale Austauschplattformen wie Twitter kommunizieren.

Die Überlegungen werden anhand der Open-Source-Software H5P verdeutlicht, die zur Gestaltung und Verbreitung von interaktiven Lehr-Lern-Materialien genutzt werden kann. In meiner Wahrnehmung ermöglicht sie mit Blick auf das Thema Offenheit die Verbindung von Weg und Ziel in besonderer Weise. Darüber hinaus bietet sie zahlreiche Möglichkeiten bei (noch) einfacher Bedienung, was sie in den vergangenen zwei Jahren weltweit vergleichsweise beliebt gemacht hat. Ich werde mit H5P als Richtschnur darauf eingehen, was OSS als Infrastruktur für die Öffnung von Lehre beitragen kann - aber auch, welche Probleme es anzugehen gilt.

\section{Die Hintergründe von H5P}

Die norwegische Regierung rief 2007 die Norwegian Digital Learning Arena (NDLA) ins Leben. Als landesweiter Zusammenschluss der Regierungsbezirke fällt ihr die Aufgabe zu, ein offenes Lernportal zu betreiben und die Herstellung von Lehr-LernMaterialien zu fördern. 2012 begann die NDLA die Suche nach einer neuen Lösung, um die bestehenden, interaktiven Inhalte zu ersetzen. Zu den Rahmenbedingungen, die eine Nähe zum Thema OER erkennen lassen, zählten unter anderem:

- Auf das bisher genutzte, aber proprietäre Format Adobe Flash sollte zugunsten des offenen Standards HTML5 verzichtet werden.

- Die neuen Inhalte sollten heruntergeladen und als Komplettpaket weitergegeben werden können.

- Die Inhalte sollten ausser einem gängigen Browser keine weitere Software voraussetzen.

- Der Quelltext sollte frei verfügbar sein, um nicht von bestimmten Entwicklerinnen und Entwicklern abhängig zu sein.

- Die Inhalte sollten von anderen weiterverwendet werden können, einschliesslich einiger Metadaten zu verwendeten Lizenzen. 
Die norwegische Firma Joubel erhielt letztlich den Auftrag, eine entsprechende Software zu entwickeln. Als Ergebnis der Bemühungen erschien Anfang 2013 die erste Fassung von H5P (https://h5p.org), die seither stetig weiterentwickelt wird.

Wie die genannten Rahmenbedingungen erkennen lassen, handelt es sich bei H5P um eine Autorensoftware, mit der interaktive Lehr-Lern-Materialien für das Web erstellt werden können. Die Bandbreite reicht von Multiple-Choice-Quizzes und Lückentexten über interaktive Videos bis zu Ausspracheübungen.

\section{Was Open-Source-Infrastruktur heute bereits zur Öffnung beiträgt}

Eine besondere OER-Tauglichkeit wurde H5P in gewisser Weise durch die Anforderungen der NDLA in die Wiege gelegt. Inhalte, die auf einer H5P unterstützenden Plattform bereitgestellt werden, lassen sich von dort prinzipiell in beliebige andere Websites einbetten. Darüber hinaus können die Inhalte aber auch inklusive der Programmlogik für die Interaktion als Paket heruntergeladen und auf anderem Wege verbreitet werden. Dieses Merkmal fällt in die Kategorie «Re-Usability». Es bezieht sich allerdings nicht nur auf die Möglichkeit der Nutzung durch andere, sondern in einigen Fällen auch durch die weitere Nutzung durch die Erstellerinnen und Entwicklern selbst: Lernmanagement-Systeme wie etwa moodle bieten ebenfalls diverse Möglichkeiten, um interaktive Inhalte zu erstellen, diese sind jedoch in der Regel an die Plattform gebunden, sollten nicht andere Standards wie SCORM unterstützt werden. Wird die Plattform gewechselt, können Lehrende die erstellten Interaktionen nicht ohne Weiteres weiterverwenden. Im schlimmsten Fall müssen sie komplett neu erstellt werden. Dank der Komplettpakete bei H5P, bestehend aus Inhalt und Programmlogik, ist er hier einfacher möglich, Bestehendes weiter zu verwenden.

Die H5P-Pakete bieten noch eine weitere Besonderheit, die der (Re-Usability) zuzurechnen ist. Nutzerinnen und Nutzer können Urheberrechtshinweise aufnehmen, die sich als Metadaten an die genutzten Medien binden lassen und auch in einer Komplettübersicht für ein Paket angezeigt werden. Weitere Unterstützung für Lehrende ist bereits in Diskussion (vgl. H5P-Forum 2017b). Dazu zählen etwa Warnungen bei Kombination von inkompatiblen Lizenzen oder das Übernehmen bestehender Lizenzinformationen beim Einbinden von Medien weiterer Dienstleister wie pixabay oder flickr.

Für Lehrende kann eine solche Unterstützung eine grosse Hilfe sein und Ängste vor den Tücken des Urheberrechts mindern. Da gerade Fragen zu diesem Thema immer wieder in der OER-Szene auftauchen und Lehrenden Sorge bereiten, kann der Infrastruktur eine gewisse Rolle bei der Öffnung von Lehrmaterial zugeschrieben werden.

Eine weitere Schwierigkeit der OER-Praxis, die immer wieder auftritt, ist das Auffinden geeigneter Inhalte («Discoverability»). Loviscach (2017, ab 14:39) etwa 
beklagt, dass es extrem aufwändig sei, etwas Passendes für die eigene Lehre zu finden. Es gäbe zwar Suchmaschinen - auch speziell für offen lizenzierte Inhalte - aber hier werde man von Inhalten regelrecht erschlagen. Loviscach hält aus seiner eigenen Praxiserfahrung heraus fest, dass es oft einfacher sei, selbst etwas Neues zu erstellen. Ziedorn, Derr und Neumann $(2013,3)$ halten zur Auffindbarkeit fest:

Um OER auszutauschen, zu finden, zu beschaffen und sie auf einer breiten Basis zugänglich zu machen, insbesondere auch über Suchmaschinen und dadurch verwenden zu können, werden für die jeweiligen Materialien Metadaten benötigt. Metadaten sind beschreibende Informationen, die einem Objekt zugeordnet sind (,Daten über Daten“). Sie helfen dabei, ein Objekt auffindbar zu machen und eindeutig identifizieren zu können.

Die bestehende Infrastruktur scheint das Repertoire an Möglichkeiten noch nicht auszuschöpfen. H5P bildet hier keine Ausnahme. Es lassen sich zwar, wie zuvor erwähnt, Angaben zum Urheberrecht ergänzen, doch sind diese nicht maschinenlesbar und nur schwerlich automatisiert ergänzbar. Des Weiteren fehlen zusätzliche, beschreibende Daten für die Inhalte selbst oder für deren vorhergesehenen Einsatzkontext, etwa Schlagwörter zu den behandelten Themen oder Klassenstufen.

Infrastrukturen können hier die Auffindbarkeit durch mehrere Mechanismen verbessern. Einerseits können sie die Fakten bereits während des Erstellungsprozesses erheben. So, wie H5P es für jedes integrierte Element ermöglicht, die Urheberrechtsinformationen anzugeben, könnten auch Metadaten erhoben werden. Im besten Fall wird dieser Vorgang (halbautomatisch) unterstützt, indem die Software die Inhalte untersucht, etwa Videos automatisch analysiert, und Vorschläge zur Verschlagwortung macht. Am Ende sollte alles in einer auf Lehrinhalte fokussierten, möglichst standardisierten und für Suchmaschinen wie Google optimierten Form ausgeliefert werden.

Alternativ bzw. zusätzlich könnte die Infrastruktur auf gesonderte, verwaltete Verzeichnisse setzen, in denen Materialien inklusive ihrer Beschreibung abrufbar sind. Solche Repositorien können gezielter auf die Bedürfnisse spezieller Nutzergruppen eingehen oder speziell auf bestimmte mediale Formen zugeschnitten sein. Gegen Ende des Jahres dürfte für H5P der sogenannte «Content Hub» erschienen sein, der diese Funktion übernimmt. Erstellerinnen und Ersteller von Inhalten können diese, wie zuvor angedeutet, in geführten Dialogen mit Schlagworten und Urheberrechtsangaben versehen. Das «Sharing) der Inhalte wird besonders einfach sein. Statt sie auf einer speziellen Plattform hochladen zu müssen, können Erstellerinnen und Ersteller dies im Erstellungsprozess durch Setzen eines Hakens erledigen. Dadurch - so die Hoffnung - könnte bei Anwenderinnen und Anwender die Bereitschaft zum Teilen ihrer Inhalte steigen. Umgekehrt können sie über den in die Software integrierten Content Hub Inhalte anderer suchen, nach verschiedenen Kriterien filtern und per Knopfdruck übernehmen. 
Die «Outreach»-Komponente, gewissermassen die Öffentlichkeitsarbeit für die eigene Auseinandersetzung mit OER, könnte sich beispielsweise direkt an das Teilen anknüpfen. Ganz wie Lernende am Ende von interaktiven Kurspräsentationen mit H5P ihre Ergebnisse auf Twitter oder Facebook teilen können, liesse sich das für die Erstellerinnen und Ersteller von Inhalten umsetzen: «Du hast schon 50 offen lizenzierte Materialien erstellt. Möchtest du die Welt das wissen lassen?»- «Soll dein Inhalt auf der OER World Map sichtbar gemacht werden?» - usw.

Der Aspekt der «Collaboration» bezieht sich bei Euler und Poupart (2018) auf das Erstellen und Anwenden der Inhalte, sowohl durch Lehrende wie durch Lernende. Gerade in Szenarien, in denen Personen zeitlich und räumlich voneinander getrennt sind, hat die Infrastruktur hier eine tragende Rolle oder macht eine effiziente Zusammenarbeit überhaupt erst möglich. Die exakten Anforderungen variieren dabei je nach den vorliegenden Rahmenbedingungen. Zwei bedeutsame Aspekte seien jedoch herausgegriffen.

Beim Erstellen von Inhalten durch mehrere Personen kann es zu Konflikten kommen, wenn an derselben Stelle Änderungen vorgenommen werden. Die Infrastruktur kann hier auf verschiedene Weise Hilfestellung bieten. Die Bandbreite reicht dabei von der Sperrung von Inhalten, solange eine Person daran arbeitet, über die möglichst zügige Synchronisation, um Konflikte frühzeitig festzustellen, bis hin zur (halb)automatischen Zusammenführung und dem Vorhalten einer Versionshistorie, um alte Fassungen zu Rate ziehen zu können. H5P bietet diesbezüglich kaum Unterstützung. Mehrere Personen können Inhalte bearbeiten und werden beim Speichern gegebenenfalls darüber informiert, wenn jemand anderes diese zeitgleich geändert hatte. Ein Überschreiben bestehender Inhalte wird dann zwar verhindert, indem in einer zweiten Fassung gearbeitet wird, aber weitere Unterstützung bei der Auflösung des Konfliktes bzw. Zusammenführung der Änderungen gibt es derzeit nicht.

Die kollaborative Nutzung von Inhalten steht infrastrukturell noch zu wenig im Fokus. Unter Interaktion wird vielfach lediglich ein Wechselspiel zwischen einer Person und einem Inhalt verstanden, sei es das Rezipieren eines Textes, das Ansehen eines Videos oder das Bearbeiten von Aufgaben. Folgt man dem, was aus bildungswissenschaftlicher Sicht schon lange bekannt ist oder dem, was jüngst als notwendige Kompetenzen für das 21. Jahrhundert diskutiert wird (vgl. P21 2015), greift dies zu kurz. In diesem Falle sollten auch Kommunikation und Kollaboration innerhalb interaktiver Lehr-Lern-Inhalte von der Infrastruktur unterstützt werden. Das gilt auch für H5P. Kooperative Inhaltstypen wie beispielsweise gemeinsame Videoannotation sind zwar angedacht, aber nicht verfügbar (vgl. H5P-Forum 2017a).

Da Eulert und Poupart (2018) ihre Einteilung lediglich auf menschliche Akteure bezogen haben, sind sie nicht auf die Kollaboration mit bzw. zwischen Infrastrukturkomponenten eingegangen. Speziell hier bietet H5P jedoch mit Blick auf OER Potenzial: Die Software verwendet die Experience API (xAPI). Dahinter verbirgt sich 
eine offene Spezifikation, mit der Datensätze über die Erfahrungen von Lernenden gesammelt, gespeichert und ausgetauscht werden können (https://experienceapi. com/overview/). H5P selbst hält diese Daten nicht vor, kann sie jedoch an jede dafür vorgesehene Gegenstelle weiterleiten.

Der naheliegende Verwendungszweck sind Learning-Analytics-Szenarien, in denen Lehrende die Erfahrungen (einer grossen Zahl) von Lernenden softwaregestützt auswerten, um daraus Erkenntnisse für die weitere Gestaltung von Lehr-Lern-Szenarien zu gewinnen. Die gewonnenen Daten könnten anderen natürlich selbst ebenfalls wieder als offene Ressource zur Verfügung gestellt werden. Weniger naheliegend, aber durch eine entsprechende Infrastruktur denkbar, ist die Ermächtigung der Lernenden selbst. Werden ihnen die von ihnen selbst erzeugten Daten über die XAPI zur Verfügung gestellt und aufbereitet, können diese reflexive Lernprozesse anregen. Mit einer entsprechenden Infrastruktur könnten Lernende sich sogar eigenverantwortlich ein Motivationssystem erstellen, das auf sie zurechtgeschnitten ist (vgl. zu Details Tacke 2016).

\section{Über freie Bildungsmaterialien hinaus}

Die vorherigen Absätze gehen schon etwas über das hinaus, was landläufig unter freien Bildungsmaterialien verstanden wird. Die Überlegungen beziehen sich auf allgemeine Ziele von Bildung, etwa die «Befähigung zu vernünftiger Selbstbestimmung» (Klafki 2007, 19). Sie beziehen sich speziell auch auf die Förderung offener Bildungspraktiken (hier Emanzipation), die in Diskussionen oft hinter der Verwendung offener Materialien zurückbleiben (vgl. Mayrberger und Hofhues 2013). Ähnlich verhält es sich bei Open-Source-Software. Sie wird häufig auf kostenlos verfügbare Programme oder frei zugängliche Standards reduziert. Die dahinter liegende Haltung gerät ins Hintertreffen.

An dieser Stelle sei betont, dass nicht jedes Open-Source-Projekt getragen ist von Werten, die auch zentral für die Bildung sind: «sharing, giving and generosity» (Wiley 2010, 20). Ist dies jedoch der Fall, ergibt die Verwendung einer entsprechend erstellten Software mindestens ein ästhetisch rundes Gesamtbild, das eingangs als "Der Weg ist das Ziel» umschrieben wurde. Im besten Fall könnte Software jedoch als Vehikel von Werten innerhalb von Gemeinschaften auch dabei helfen, diese in die Bildungswelt zu tragen (vgl. Coleman und Hill 2005). Aus diesem Grund werden folgend einige organisatorische Aspekte von Open-Source-Projekten beleuchtet, die einen Beitrag zu Open Education leisten können.

Das offensichtlichste und namensgebende Merkmal lautet: Der Quelltext ist frei verfügbar. Er lädt dazu ein, studiert und nach den eigenen Vorstellungen verändert zu werden (Emanzipation). Das Spektrum der Möglichkeiten reicht dabei von einfachen Anpassungen, etwa dem Ändern von Farben oder der Übersetzung von 
Bildschirmtexten in andere Sprachen bis hin zur Ergänzung fehlender Funktionen. Nutzerinnen und Nutzer der Software sind dazu eingeladen, ihre passive Rolle zu verlassen und dabei zu helfen, die Software voranzubringen (Partizipation).

In der Praxis herrscht bezüglich der Entwicklung der Software oft der Trugschluss, sie sei lediglich Programmiererinnen und Programmierern vorbehalten. Es gibt allerdings zahlreiche Möglichkeiten, sich einzubringen (vgl. Tacke 2017). Wer einen Fehler findet oder vermutet, kann inn melden. Wer Funktionen vermisst oder geändert wünscht, kann dies vorschlagen. Viele Projekte, wie auch H5P, nutzen dafür eigens eingerichtete Foren. Anwenderinnen und Anwender können ihre Ideen direkt mit den Entwicklerinnen und Entwickler diskutieren.

Anders als bei geschlossenen Produkten besteht hier die Chance, durch Beteiligung den Problemen «palliativer Technik» entgegenzuwirken. Krommer (2017) versteht darunter technische Massnahmen wie Apps oder Whiteboards, die das traditionelle Bildungssystem mit neuen digitalen Instrumenten fortführt statt es grundlegend zu verändern. Der laute Ruf nach Digitalisierung und die einfache Handhabung von H5P könnten dazu führen, über Gebühr beispielsweise Multiple-Choice-Quizzes statt sinnvollerer Interaktionen zu verwenden. Rosa (2017) bezeichnet dies als «Toolifizierung» bzw. «Quizzifizierung».

Viele grössere Open-Source-Projekte verwenden zudem spezielle Software zum Managen der Aufgaben der beteiligten Personen. Es lässt sich darin nachvollziehen, wer gerade an welcher Funktion arbeitet, welche Probleme auftreten, usw. je nach Projekt ist auch dieses System für jeden einsehbar (Transparenz). Auch das H5P-Projekt gewährt diesen Einblick.

\section{Fazit}

Die technische Infrastruktur, die zum Erstellen, Teilen und Remixen von freien Bildungsmaterialien genutzt wird, kann erheblichen Einfluss auf die Gestaltung derselben haben und verdient eine stärkere Betrachtung als bisher. Die technische Infrastruktur ist nicht nur ein Werkzeug, das didaktischen Überlegungen Wirkung verleiht, sondern gleichsam unterschwelliges Vehikel für zu transportierende Werte. Da OpenSource-Software viele Werte mit der OER-Bewegung teilt, sollte ihr trotz der immer wieder auftretenden Unbequemlichkeiten im Vergleich zu sunfreier) Software der Vorzug gegeben werden. 


\section{Literatur}

Coleman, Enid Gabriella, und Benjamin Hill. 2005. «The Social Production of Ethics in Debian and Free Software Communities: Anthropological Lessons for Vocational Ethics». In Free/Open Software Development, herausgegeben von Stefan Koch, 273-295. Hershey: Idea Group Publishing.

Dueck, Gunter. 2009. «Culture Technologies - Dreimal mehr in Herz und Kopf!». InformatikSpektrum 32 (1): 65-69. https://doi.org/10.1007/s00287-008-0311-8.

Euler, Ellen, und Raoul Poupart. 2018. «Spectrum of Open Education Practices (Version 1.0.)». https://doi.org/10.5281/zenodo.1164799.

H5P-Forum. 2017a. «Competitive/Cooperative learning with H5P?». https://h5p.org/ node/81653.

H5P-Forum. 2017b. «Improve H5P regarding handling copyright». https://h5p.org/ node/109944.

Klafki, Wolfgang. 2007. Neue Studien zur Bildungstheorie und Didaktik. Zeitgemäße Allgemeinbildung und kritisch-konstruktive Didaktik. 6. Aufl. Weinheim: Beltz.

Krommer, Axel. 2017. «Notwendige Neologismen: „Palliative Technik“». https://axelkrommer. com/2017/10/01/notwendige-neologismen-palliative-technik.

Loviscach, Jörn. 2017. «Digitale Medien in der Hochschullehre, ein Experimentierfeld». https:// youtu.be/djyjRT8vFHM?t=14m39s.

Mayberger, Kerstin, und Sandra Hofhues. 2013. «Akademische Lehre braucht mehr „Open Educational Practices“ für den Umgang mit „Open Educational Resources“ - ein Plädoyer». Zeitschrift für Hochschulentwicklung ZFHE 8 (4): 56-68. https://doi.org/10.3217/zfhe-804/07.

Muuß-Merholz, Jöran. 2014. «OER-Matrix: eine Systematik zu den Ausprägungen von Open Educational Resources». http://pb21.de/2014/12/oer-matrix/.

Muuß-Merholz, Jöran. 2017. "Offen ist, was Zugang schafft! Oder: Warum Google Docs für OER wichtiger als Libre Office ist». https://open-educational-resources.de/offen-ist-waszugang-schafft-oder-warum-google-docs-fuer-oer-wichtiger-als-libre-office-ist.

P21. 2015. «P21 Framework Definitions». http://www.p21.org/storage/documents/docs/P21_ Framework_Definitions_New_Logo_2015.pdf.

Rosa, Lisa. 2017. «Welche „digitale Bildungsrevolution“ wollen wir?». https://prezi.com/2 a0meyx19yc/welche-digitale-bildungsrevolution-wollen-wir.

Stalder, Felix. 2016. Kultur der Digitalität. Berlin: Suhrkamp.

Tacke, Oliver. 2016. «if ( xAPIcall ) \{ myChoice(); \}». https://www.olivertacke.de/2016/09/08/ if-xapicall-mychoice.

Tacke, Oliver. 2017. «Open-Source-Software voranbringen - auch wenn du nicht programmieren kannst!». https://www.olivertacke.de/2017/05/19/open-source-software-voranbringen-auch-wenn-du-nicht-programmieren-kannst. 
Wiley, David. 2010. «Openness as Catalyst for an Educational Reformation» EDUCAUSE Review 45 (4): 14-20. https://er.educause.edu/ /media/files/article-downloads/erm1040.pdf.

Ziedord, Frauke, Derr, Elena, und Janna Neumann. 2013. «Metadaten für Open Educational Resources (OER). Eine Handreichung für die öffentliche Hand, erstellt von der Technischen Informationsbibliothek (TIB)». https://www.pedocs.de/volltexte/2013/8024/pdf/ TIB_2013_Metadaten_OER.pdf. 